\title{
Electrodynamics of the vertical coupling processes in the atmosphere-ionosphere system of the low latitude region
}

\author{
M. A. Abdu and C. G. M. Brum \\ Instituto Nacional de Pesquisas Espaciais-INPE São José dos Campos, SP, Brazil \\ (Received October 9, 2007; Revised February 1, 2008; Accepted February 1, 2008; Online published May 14, 2009)
}

\begin{abstract}
Dynamical, electro-dynamical and electrical coupling processes originating from upward propagation of atmospheric waves, and magnetosphere-ionosphere interaction are responsible for the large degree of variabilities observed in the low latitude ionosphere. One of the most outstanding aspects of its phenomenology is related to the sunset electrodynamical processes responsible for the evening enhancements in zonal and vertical electric fields and the associated spread of $F$ /plasma bubble irregularity development. Recent observational results have provided evidence of significant contribution to their quiet time variability arising from thermospheric wind patterns, upward propagating planetary waves and possibly sporadic $E$ layers. This paper provides an overview and some new results on planetary wave coupling with the equatorial $F$ region, the $E$ layer conductivity as key connecting mechanism, a possibly interactive role by sporadic $E$ layers, and the resulting day-to-day variability in the evening prereversal electric field enhancements with consequences on spread $F$ development.
\end{abstract}

Key words: Vertical coupling, atmosphere-ionosphere, spread $F$, planetary waves, sporadic $E$ layers, prereversal electric field.

\section{Introduction}

Coupling processes involving different external forcing mechanisms are responsible for the major phenomenology and the day-to-day variabilities of the equatorial atmosphere ionosphere system. Forcing by vertical coupling through upward propagating atmospheric waves, as tidal modes, gravity waves and planetary waves etc, is a well recognized source of variability, (e.g., Forbes, 1996; Pancheva et al., 2002; Abdu et al., 2006a, b; Fukao, 2006; Ogawa et al., 2006), while enhanced ionosphere-magnetosphere coupling processes during episodes of space weather disturbances and changing solar radiation flux provide another important source of the variabilities. Extensive investigations have been conducted in recent years aimed at gaining a better knowledge of these forcing mechanisms vis-vis the ionospheric and atmospheric responses in quantitative as well as qualitative terms. Such knowledge is necessary for developing predictive capabilities on such variabilities. While we have a good deal of knowledge on the response characteristics of the low latitude ionosphere-thermosphere system to forcing from interplanetary and magnetospheric processes, our knowledge of the vagaries of the system arising from the vertical coupling processes involving wave disturbances originating from lower atmosphere is very limited indeed. Whether they originate from the lower atmosphere or by external forcing from above, the variabilities occurring during the evening to post-sunset and night hours have

Copyright (c) The Society of Geomagnetism and Earth, Planetary and Space Sciences (SGEPSS); The Seismological Society of Japan; The Volcanological Society of Japan; The Geodetic Society of Japan; The Japanese Society for Planetary Sciences; TERRAPUB. specific significance, the main reason being that sunset electrodynamic processes play a fundamental role in shaping the post-sunset equatorial ionization anomaly and plasma bubble irregularity distribution that impact on diverse space application systems. This paper will focus on some aspects of the vertical coupling processes highlighting the consequences on the sunset electrodynamics and related problems, paying particular attention to the possible causes of the widely observed day-to-day variability in the evening prereversal electric field enhancement (EPE) and equatorial spread $F(\mathrm{ESF}) /$ plasma bubble development conditions.

The entire vertical coupling process can be seen as a twostage process: (a) dynamic processes by which the wave energy from the lower heights/regions (troposphere and stratosphere) propagates to higher levels in middle atmosphere and lower thermosphere (MLT) regions (including the lower $E$-region heights); (b) the subsequent sequences in which the electrodynamic processes take over at height levels near and above the $E$ layer extending well into the $F$ region. In this paper we intend to briefly evaluate some recent results concerning mainly the aspect (b) and discuss their implications further, based on some new results and interpretations, to lead to a better understanding of the quiet time variability of the equatorial $F$ region vertical plasma drifts and ESF irregularity generation processes. The possible interactive roles of the evening sporadic $E$ layers in these processes will also be discussed. The results are organized into sections 'Some considerations on sunset electrodynamics'; 'Planetary wave effects on evening $F$ layer heights, prereversal electric field enhancement and ESF'; 'Sporadic $E$ layers and EPE: interactive roles'. The results are then dis- 


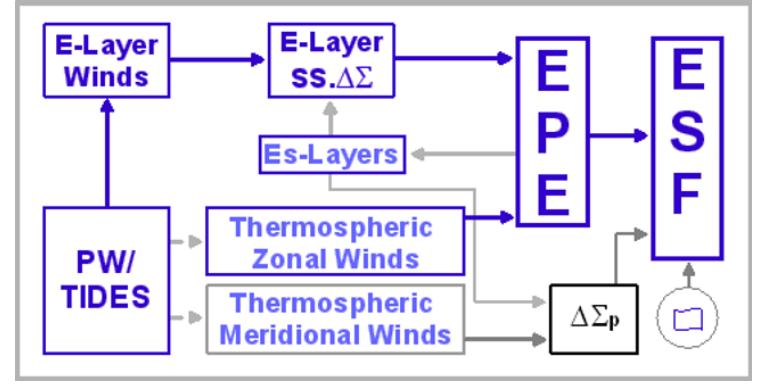

Fig. 1. A scheme of the processes leading to the development of the evening prereversal electric field (EPE) and equatorial spread $F$ (ESF).

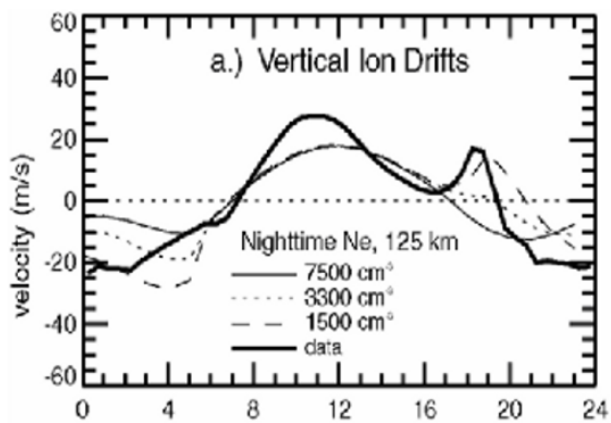

Fortaleza - June 2001
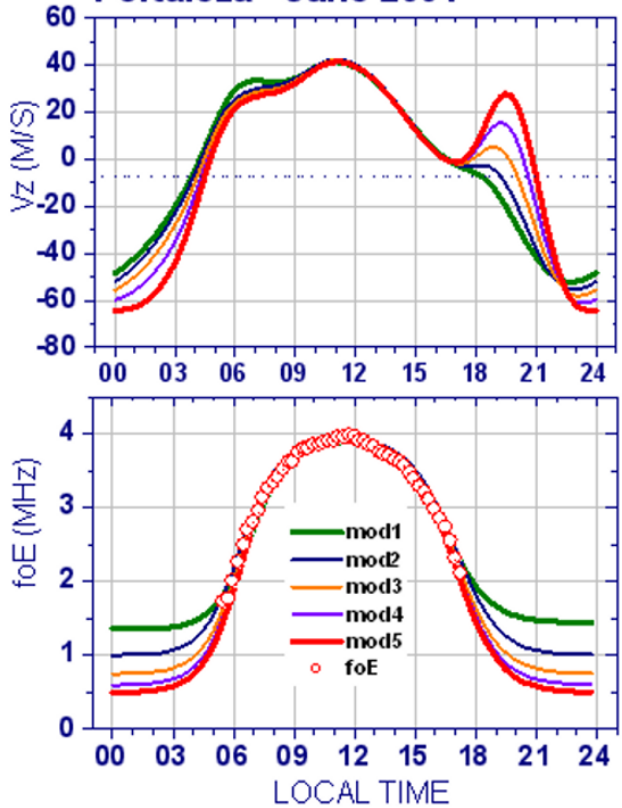

Fig. 2. F region vertical drift as modeled by the TIEGCM (Fesen et al., 2000) (upper panel) and that modeled by the formulation by Heelis et al. (1974). The amplitude of the EPE increases with decrease in night-time electron density as modeled by the TIEGCM and by the increase in the sunset conductivity/electron density local time/longitudinal gradient as modeled by the Heelis et al. (1974) formulation.

cussed and a conclusion drawn.

\section{Some Considerations on Sunset Electrody- namic Processes}

The ionospheric dynamo driven by the wind systems at $E$ - and $F$-layer heights, in the presence of the conductivities and the geomagnetic field, are responsible for the major phenomenology of the low latitude region. With the decay of the $E$ layer conductivities, the $F$ layer dynamo electric fields begin to dominate towards the evening and night hours. The immediate manifestation is the development of the evening prereversal electric field that develops under the action of an eastward thermospheric zonal wind in the presence of the longitudinal/local time gradient in the integrated $E$ layer Hall and Pedersen conductivities across the sunset terminator (Rishbeth, 1971; Heelis et al., 1974; Batista et al., 1986). A scheme of the processes leading to the development of the EPE is presented in Fig. 1 wherein the longitudinal/local time gradient in the integrated conductivity at sunset hours denoted as $S S . \Delta \Sigma$ is shown acting together with the thermospheric zonal winds as the basic cause of the EPE development. We may point out that the mechanisms widely believed to operate in the development of EPE involve longitudinal gradients in the Pedersen conductivity that works for the $F$ layer dynamo, as first explained by Rishbeth (1971). Farley et al. (1986) have discussed the processes of $F$ layer dynamo electric field interacting with conjugate $E$ regions where the east-west Hall current interruption at the terminator could result in zonal electric field enhancement that constitutes the EPE. Haerendel and Eccles (1992) discussed the problem of EEJ current divergence at the sunset contributing to the EPE (see also, Eccles (1998) for an idea on the relative importance of these contributions in EPE development). Thus, the longitude/local time gradients in both the Pedersen and Hall conductivities are represented in the parameter $S S . \Delta \Sigma$ indicated in Fig. 1. The zonal electric field/vertical plasma drift pattern as modeled by the Thermosphere-IonosphereElectrodynamics General Circulation Model (TIEGCM) by Fesen et al. (2000) and that modeled of Batista et al. (1986) using the formulation by Heelis et al. (1974), as adapted from Abdu et al. (2004), are presented in Fig. 2. Note that the amplitude of the EPE increases with decrease of night time electron density in the TIEGCM and equivalently by the increase in the negative gradient in the $E$ layer density/integrated conductivity at sunset as modeled using the formulation by Heelis et al. (1974). The enhancement of the eastward electric field of the EPE is followed by an enhancement in westward electric field (downward drift). The post-sunset horizontal plasma shear flow (Tsunoda et al., 1981), characterized by a westward flow in the bottom-side $F$ region below $\sim 300 \mathrm{~km}$ with eastward flow just above this height, constitute, together with the upward and downward flow just described above, the plasma vortex flow of which an example is shown in Fig. 3, (left panel) taken from Kudeki and Bhattacharyya (1999). A vertical cut through the vortex center produces the height variation of the vertical electric field near 19 LT (right panel) that has important consequences on the sporadic $E$ layer development at offequatorial and low latitudes. There is an interactive role between the EPE and $E_{\mathrm{s}}$ layers that occurs when these layers are present at the footprints of the field lines participating in EPS development. Recent observational results from inosondes/digisonds in Brazil have shown that the development of $E_{\mathrm{s}}$ layers within appropriate latitudinal proximity to the dip equator can be disrupted under the action of the vertical electric field associated with the EPE development (Abdu et al., 2003; Carrasco et al., 2007). This aspect 
will be pursued later in this paper. On the other hand, the integrated conductivity of a sporadic $E$ layer could potentially negatively influence EPE development, as shown by model calculations by Carrasco et al. (2005). This EPE$E_{\mathrm{s}}$ layer interactive role is also depicted in Fig. 1 (see also the sketch in Fig. 11). Also shown in this figure is the connection among the upward propagating planetary waves (PWs), the tidal modes and the PW-modulated tidal modes that could modify the $E$ layer wind system (Pancheva et al., 2003; Haldoupis et al., 2004) with possible effects on the $E$ layer conductivity sunset gradient leading to EPE modification (Abdu et al., 2006a), as will be discussed in more detail later. The rapid uplift of the $F$ layer under the action of the EPE is known to be primarily responsible for the development of the spread $F$ /plasma bubble irregularities of the post-sunset equatorial ionosphere. The steep bottomside density gradient of the rising $F$ layer becomes unstable to density perturbations, leading to instability growth by the Rayleigh-Taylor (R-T) mechanism. The requirement of a seeding source is also indicated in Fig. 1. The instability growth by the R-T mechanism involves non linear rise up of the rarified plasma of the $F$ layer bottom side to the topside ionosphere in the form of plasma depleted flux tubes/plasma bubbles, with the associated cascading process within the bubbles leading to irregularity formation in scale sizes ranging from a few centimeters to hundreds of kilometers (Haerendel, 1973). The entire process constitutes an ESF development. Meter-scale irregularity generation has been shown to occur close to or before the terminator passage at the apex of the $F$ region field line, as diagnosed by the Equatorial Atmosphere Radar (EAR) in Indonesia (Yokoyama et al., 2004) and the irregularity structures move eastward (Fukao et al., 2006). The role of a thermospheric meridional (or a trans-equatorial) wind is to enhance the field line integrated conductivity that could retard the ESF development (Maruyama, 1988; Abdu et al., 2006c), which could in turn diminish the intensity and limit the spectral extension of the secondary irregularities. The ESF irregularities present a large degree of variability on seasonal, day-to-day and shorter terms. While the causes of the seasonal variation are fairly well known (e.g. Abdu, 2001), those of the day-to-day and shorter term variabilities are far from being identified due to our lack of detailed knowledge on the interdependent variability of the ambient parameters that control ESF development. One of the sources of such variability resides in what appears to be inherent in the nature of the seeding mechanism. In this respect, it is still being debated whether the seeding mechanism originates from a remote gravity wave source and/or a recently proposed local instability growth by velocity shear mechanism at the $F$ layer bottom-side (Hysell and Kudeki, 2004). Also under discussion is the possible role of a sporadic $E$ layer instability mechanism for initiating the conditions for ESF development (Tsunoda, 2007). More recently, an important source of the ESF day-to-day variability has been shown to arise from atmosphere-ionosphere coupling processes involving vertical propagation of planetary waves (Abdu et al., 2006a). Ionosphere-magnetosphere coupling processes under magnetically disturbed conditions are also known to be an important source of ESF variability (Sastri et al., 1997; Abdu et al., 2003).

\section{Planetary Wave Effects on Evening $F$ Layer Heights, EPE and ESF}

Vertical coupling processes involving PWs are believed to play a significant role in the day-to-day variabilities widely observed in important parameters of the equatorial and low latitude ionosphere. Planetary waves of quasi 2day and 3- to 5-day periodicities in equatorial mesospheric winds were reported by Gurubaran et al. (2001) and Vincent (1993), respectively, and in mesospheric airglow intensity by Takahashi et al. (2005). Planetary wave scale oscillations of different periods have also been identified in the equatorial electrojet current (EEJ) strength (Forbes and Leveroni, 1992; Forbes, 1996; Abdu et al., 2006b) and, more recently, in mesopause temperature and EEJ strength (Vineeth et al., 2007), equatorial ionization anomaly (Chen, 1992) and equatorial $F$ layer height and vertical plasma drift (Takahashi et al., 2005; Abdu et al., 2006a, b). The influence of the PWs on the sunset electrodynamics can result in significant modifications in the intensity of the evening prereversal electric field enhancement and the post sunset $F$ layer heights and, hence, play an important role in the widely observed day-to-day variability in the equatorial spread $F$ /plasma bubble irregularity generation, which is primarily driven by the EPE. Recent analysis has provided evidence of a direct connection between the PW manifestations in mesospheric winds and the EPE with consequent variations in the equatorial spread $F$ intensity/occurrence (Abdu et al., 2006a). Figure 4 shows (top panel) the peak evening $F$ layer vertical drift $\left(V_{\mathrm{zp}}\right)$ over the equatorial and low latitude sites, Cachimbo $\left(9.47^{\circ} \mathrm{S}, 54.83^{\circ} \mathrm{W}\right.$, dip angle: $\left.-3^{\circ}\right)$ and Campo Grande $\left(20.44^{\circ} \mathrm{S} ; 54.64^{\circ} \mathrm{W}\right.$; dip angle: $-22^{\circ}$ ), respectively, in Brazil, as obtained during the October-December 2002 COPEX campaign (Abdu et al., 2007). The $V_{z}$ was calculated from the time rate of change of the $F$ layer height (the mean of the $d h F / d t$ calculated at the plasma frequencies of 4 and $5 \mathrm{~Hz}$ ), which is a realistic vertical drift for $h F \geq 300$ (Bittencourt and Abdu, 1981). There is a large difference in the $V_{\mathrm{zp}}$ amplitudes at the two stations, the amplitude being significantly smaller over Campo Grande than over Cachimbo. The implications of these have been discussed in Abdu et al. (2007). The important point to note is that there are large day-to-day variations in the $V_{\text {zp }}$ that are mostly in-phase at the two sites. While some of these variations could be caused by magnetic activity and solar flux variations (plotted in the lower three panels), there are variations of large amplitudes (such as those during the hatched intervals in Fig. 4) that are not possibly caused by the magnetic activity, which was quiet during these periods, or by the variations in $F_{10.7}$. Some of these variations have been shown to be associated with upward propagating planetary waves. Figure 5 shows the $V_{\text {zp }}$ values (top panel) as in Fig. 4 and their wavelet power spectra (lower two panels) over the two sites. They appear well correlated at the two stations, except for some possible local effects at the two locations separated in North-South by $\sim 1200 \mathrm{~km}$. The lower four panels present the wavelet power spectra of mesospheric zonal and meridional winds at $95 \mathrm{~m}$ and $100 \mathrm{~m}$ over Cachoeira Paulista $\left(22.6^{\circ} \mathrm{S}, 315^{\circ} \mathrm{E}\right.$; 


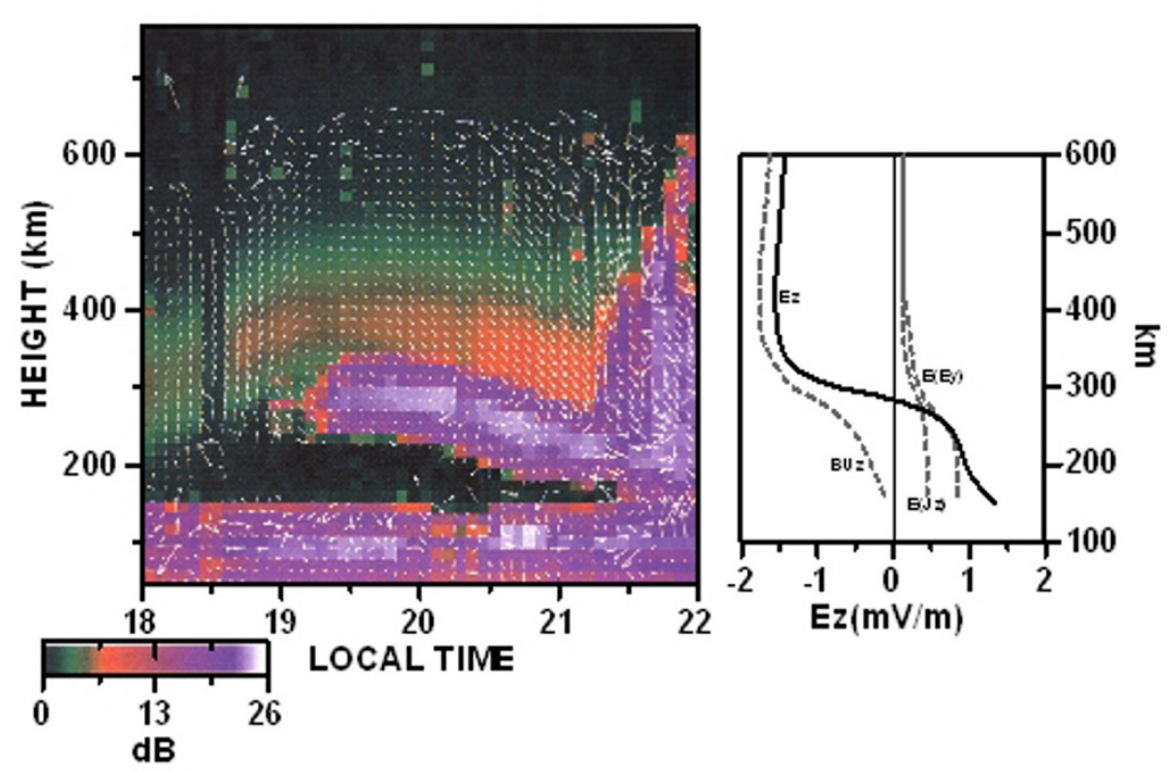

Fig. 3. Left panel A drift and backscattered power map for September 17, 1996 (sunset time 18:03 LT, $K_{\mathrm{p}}=4-$, 2-). Data quality are poor above $\sim 500 \mathrm{~km}$ as a result of interference from JRO ionosonde. (Kudeki and Bhattacharyya, 1999). Right panel A vertical profile of the vertical electric field from Haerendel et al. (1992), which resembles the electric field to be obtained from a vertical cut of the vortex flow at $\sim 19: 20$ LT in the left panel. Note that the height axes of the two panels do not match, but the height of zero $E_{\mathrm{z}}$ is about the same in both panels, which is near $300 \mathrm{~m}$.

dip angle: $-32^{\circ}$ ). (For details on the meridional and zonal wind measurements by meteor radar, see Hocking et al., 2001). We may note in Fig. 5 the presence of oscillation periods in the power spectra, ranging from $\sim 3$ to 7 days, during the interval from day 305 to day 320, that are common in the $V_{\mathrm{zp}}$ and mesospheric winds. None of the major periodicities in the $V_{\text {zp }}$ or in the mesospheric winds appears to be related to those in the magnetic or solar flux indices, as can be verified from the wavelet power spectra of these indices plotted in Fig. 6. Therefore, the day-to-day variations in the evening prereversal electric field do seem to contain a component driven by planetary waves. A wave decomposition analysis for this case by Abdu et al. (2006a) showed dominance of 4- to 5-day and 7-day periods in both the $V_{\text {zp }}$ and mesospheric winds that presented a downward phase propagation similar to that observed by Pancheva et al. (2003) from an analysis of sporadic $E$ layer features and mesospheric winds.

Ultra Fast Kelvin (UFK) waves trapped in the equatorial latitude propagating from troposphere to ionospheric heights have recently been shown to cause significant modulation in the mesospheric winds, as measured by meteor radars and in post sunset $F$ region parameters $h^{\prime} F$ and $f_{\mathrm{o}} F_{2}$, measured by ionosonde (Takahashi et al., 2007). Figure 7 shows band-pass filtered wave oscillations of 3.5-day period in $f_{\mathrm{o}} F_{2}$ (top panel) and $h^{\prime} F$ (middle panel) over Fortaleza and in the zonal wind at $90 \mathrm{~m}$ (bottom panel) over Cariri $\left(7.4^{\circ} \mathrm{S}, 36.5^{\circ} \mathrm{W}\right)$. The ionospheric data correspond to $21 \mathrm{~T}$ (one data point per day), whereas the zonal winds are hourly mean values. The dominant oscillations in all these parameters that can be noted around day 66 presented downward phase propagation with a vertical wavelength of $\sim 40 \mathrm{~km}$. From the propagation characteristics of these waves as diagnosed from measurements at widely separated

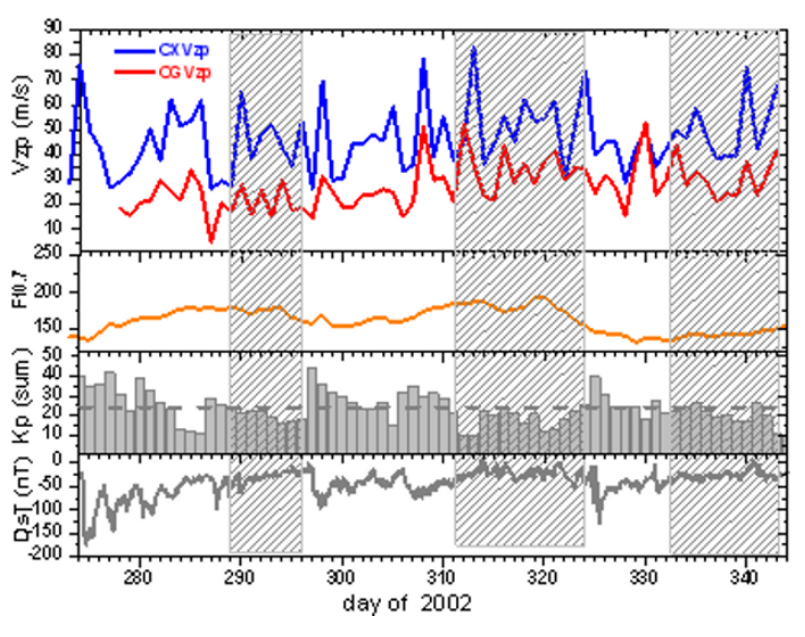

Fig. 4. The $V_{\mathrm{zp}}$ values for Cachimbo and Campo Grande plotted during the COPEX campaign days from 270 to 343 (top panel). The lower three panels show the solar flux $F_{10.7}$, the $K_{\mathrm{p}}$ and the $D_{\text {st }}$ values for this period. Intervals of magnetically quiet days are shown hatched.

longitudes and latitude, the UFK wave characteristics of these oscillations were identified. The upward propagation of these waves to ionospheric height, causing corresponding oscillations in the post-sunset $F$ region densities and heights that are controlled by the EPE, was also verified.

These results demonstrate the existence of a strong vertical coupling through upward propagating planetary waves leading to day-to-day oscillations in mesospheric winds, EPE and post-sunset $F$ region parameters. A possible electrodynamic coupling mechanism connecting these oscillations will be addressed in the discussion section.

It is well known that the EPE and the resulting $F$ layer 

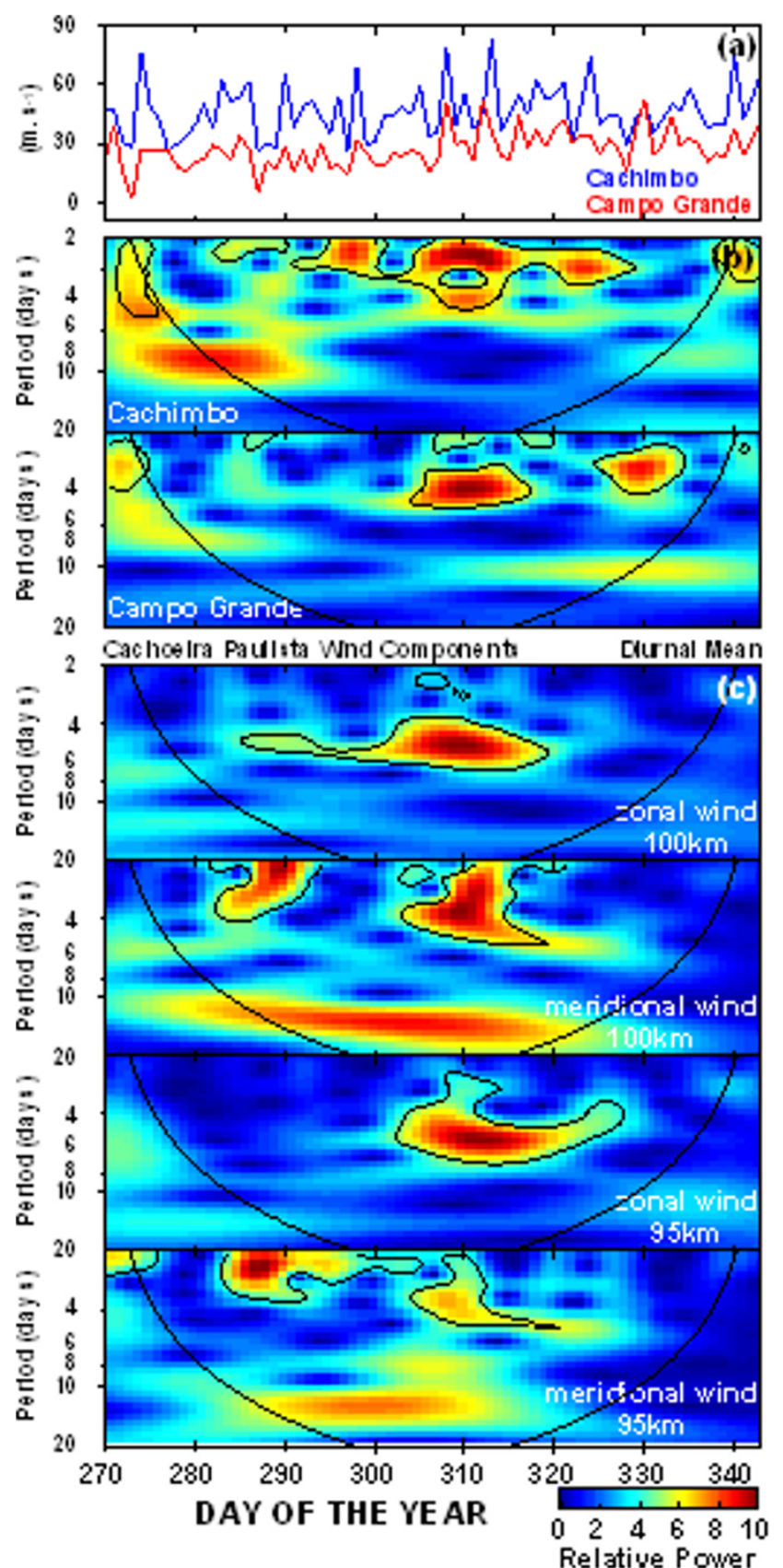

Fig. 5. (a) Vertical drift velocity $\left(V_{\mathrm{zp}}\right)$ variations over $\mathrm{CX}$ and $\mathrm{CG}$ for the period October-December 2002 (top panel) (b) Morlet wavelet power spectral distribution of $V_{\text {zp }}$ oscillations over the two stations (c) Wavelet spectral distribution of the daily means of mesospheric zonal and meridional winds at $100 \mathrm{~m}$ over Cachoeira Paulista (the two upper panels) and at $95 \mathrm{~m}$ (the two lower panels). The periods within the black line contours have $95 \%$ confidence level.

height rise are major causes for the development of the postsunset ESF irregularities (Farley et al., 1970; Abdu et al., 1983; Fejer et al., 1999). Thus, a major consequence of the PW oscillations in the EPE is its contribution to the dayto-day ESF variability widely observed under magnetically quiet conditions (Abdu et al., 2006a, b). The statistical association between the $V_{\mathrm{zp}}$ over Cachimbo and the ESF intensity over the same site as well as over two low latitude sites CG and CP are presented in Fig. 8 for the period October to December 2002 (see also Abdu et al., 2007). (The spread $F$ (SF) intensity is represented in terms of the increasing degree of range spreading indexed as 1, 2, 3, for details, see Abdu et al., 1983). At Cachimbo, where the SF first develops and the irregularity strength appears to reach the well-developed stage typically within about $30 \mathrm{~min}$ or more, the spread $F$ occurrence between 18 and $20 \mathrm{~T}$ is considered. At Campo Grande and Cachoeira Paulista where the SF occurs with some time delay (with respect to its occurrence over dip equator) due to the plasma bubble vertical growth time, the event intensities from 18 to 24 LT are considered. We note from the scatter plots that over Cachimbo the bottom side SF does not occur for $V_{\mathrm{zp}}$ less than a threshold value of $25 / \mathrm{s}$. The threshold values of $V_{\mathrm{zp}}$ for varying degrees of topside bubble developments, as indicated by the SF over CG and CP, are around 32-35 m/s. These threshold values are generally consistent with previous results (Fejer et al., 1999; Abdu et al., 2006c). Overall, there is a trend of the SF intensity to increase with increasing $V_{\text {zp }}$ over all the sites. The degree of scatter in the SF intensity for a given $V_{\mathrm{zp}}$ amplitude indicates additional factors shown in Fig. 1 that control the SF intensity, including (1) the amplitude of the seed perturbation (such as gravity waves) needed for the R-T instability mechanism to operate and (2) field line integrated conductivity depending upon thermospheric meridional/trans-equatorial winds (Maruyama, 1988; Abdu, 1997; Mendillo et al., 2001) of which some recent results have been presented by Abdu et al. (2006c, 2007). A discussion on the role of gravity waves in seeding the ESF is beyond the scope of this paper (but see the overview paper by Fritts et al. (2009) in this issue). The competitive roles played by items (1) and (2) in the ESF growth conditions can cause some ambiguity in the identification of the ESF variability as arising from a specific planetary wave episode whose effects on the EPE is more readily identifiable.

\section{Evening Prereversal Electric Field Enhance- ment and Sporadic $E$ Layers: Interactive Roles}

When investigating the PW effects on ESF operating through the EPE (Fig. 1) it is necessary to examine some additional factors that affect both the EPE and ESF and the recently proposed interactive connection between the EPE and the evening sporadic $E$ layers (Abdu et al., 2003; Carrasco et al., 2005, 2007) is a case to be considered. Abdu et al. (2003) showed that sporadic $E$ layer formation during post-sunset hours can be interrupted/disrupted under the development of the EPE of sufficient intensity, meaning that the EPE of a smaller than a critical value does not interrupt the $E_{\mathrm{s}}$ layer development. Several examples of such cases were presented by Abdu et al. (2003) and Carrasco et al. (2007). An interesting case of $E_{\mathrm{s}}$ layer disruption is presented in Fig. 9(a) which shows the disruption of a sequential/descending $E_{\mathrm{s}}$ layer at a height of $\sim 120 \mathrm{~km}$ at $\sim 18$ LT when the $F$ layer base height $\left(h^{\prime} F\right)$ in its rapid ascent was at $\sim 300 \mathrm{~m}$ and when the vertical drift was $>37 / \mathrm{s}$. The wind-shear responsible for the $E_{\mathrm{s}}$ layer formation appears to have persisted for an extended duration so that the restructured $E_{\mathrm{s}}$ layer at $21 \mathrm{LT}$ continued its descent at the descent rate of the wind shear nodal point until past midnight. More examples of the $E_{\mathrm{s}}$ layer disruption associ- 


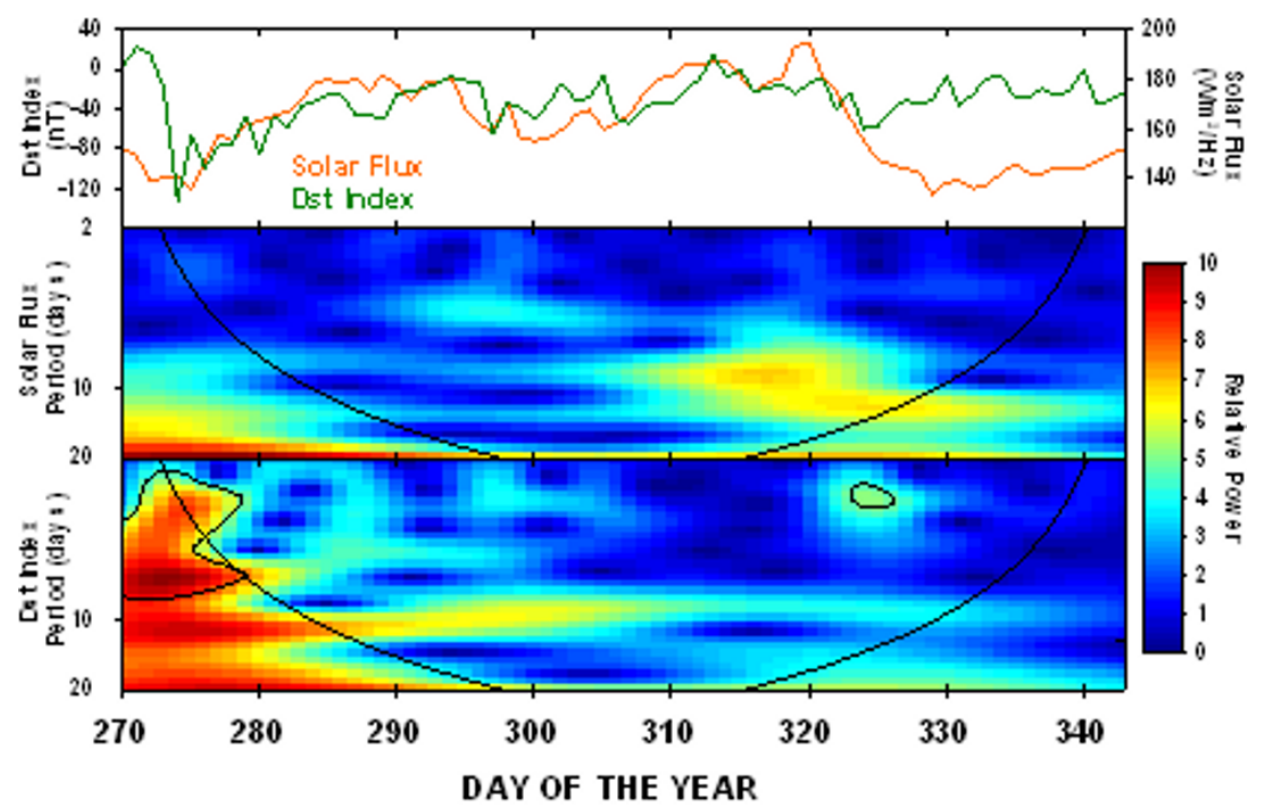

Fig. 6. Wavelet power spectra of the $F_{10.7}$ and $D_{\text {st }}$ parameters during the same period as that of the data analyzed in Fig. 5 .

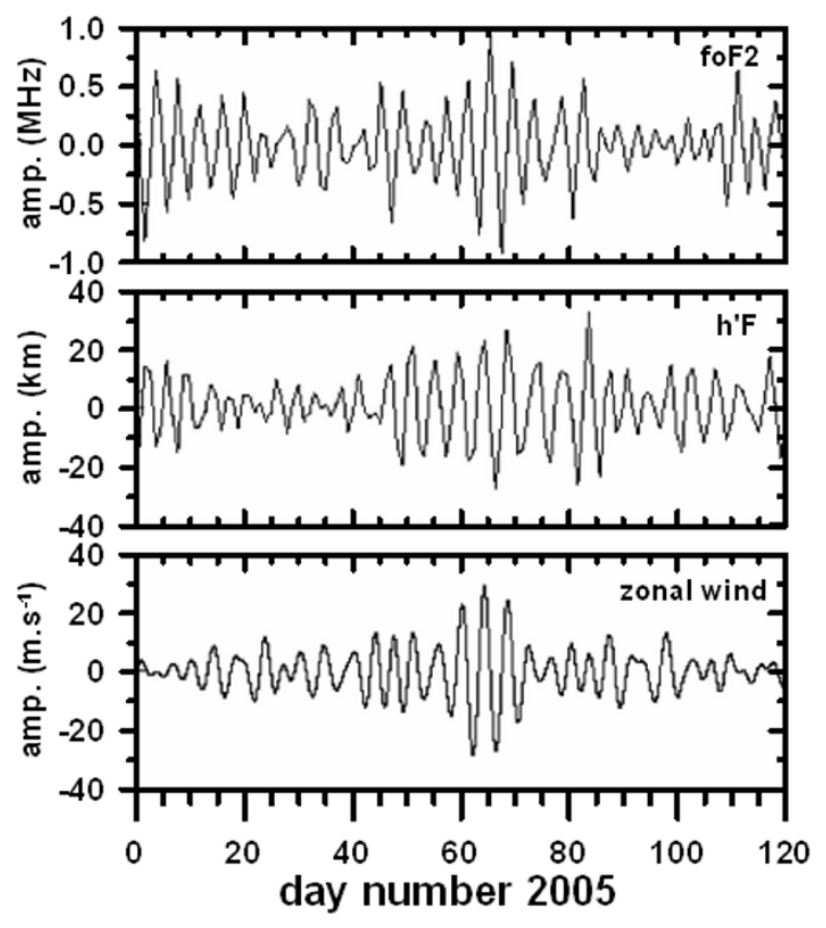

Fig. 7. Amplitude and phase of the 4 \pm 1 -day filtered oscillations of Fortaleza $f_{\mathrm{o}} F_{2}$ (top), $h^{\prime} F$ (middle), and Cariri zonal wind at $90 \mathrm{~m}$ (bottom) during the period from January 1 to April 30, 2005 (Takahashi et al., 2007).

ated with the EPE are presented in Fig. 9(b) (taken from Carrasco et al., 2005). The examples presented for a set of days in June 2001 show that for a large $F$ layer uplift (left panel) corresponding to a mean vertical velocity of $\sim 35 \mathrm{~m} / \mathrm{s}$, a disruption of the ongoing $E_{\mathrm{s}}$ layer formation occurred, whereas for another set of days (right panel), for which the mean vertical drift was $\sim 15 \mathrm{~m} / \mathrm{s}$, the ongoing $E_{\mathrm{s}}$ layer was not disrupted. Such a result was explained by Abdu et al. (2003) in terms of the effect of the verti-

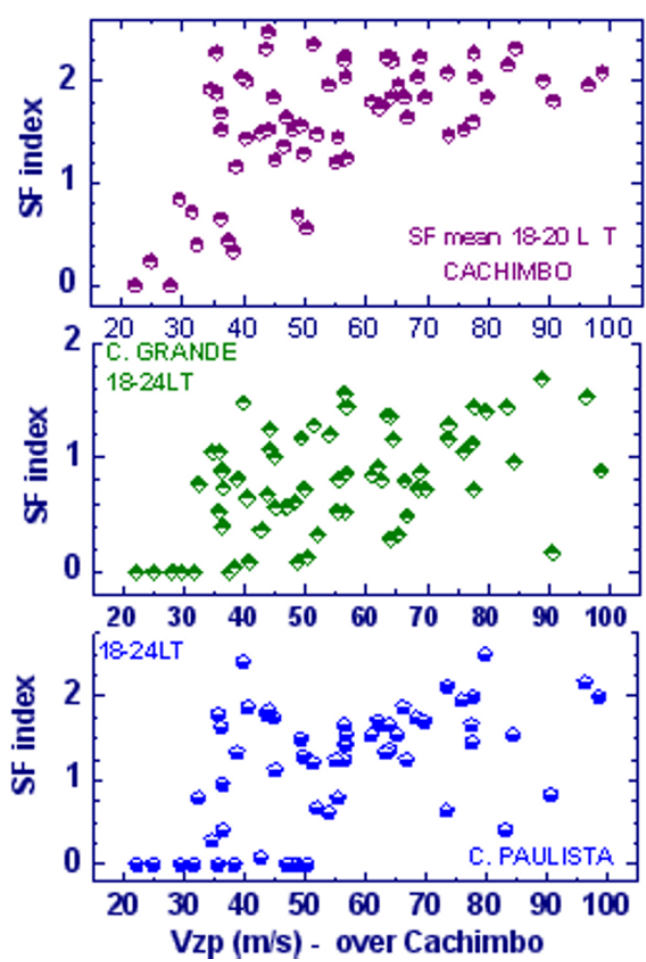

Fig. 8. The evening vertical drift velocity peak $\left(V_{\mathrm{zp}}\right)$ plotted versus the spread $F$ intensity indexed by numbers indicating the range spreading in hundreds of kilometers: ' 1 ' indicates $100 \mathrm{~km}<$ spread range $\leq 200 \mathrm{~km}$; '2' stands for $200 \mathrm{~km}<$ spread range $\leq 300 \mathrm{~km}$, and ' 3 ' stands for spread range $>300 \mathrm{~km}$.

cal electric field, associated with the EPE development, in causing vertical plasma transport at $E$ layer heights. Depending upon whether directed upward or downward, such an electric field could retard or enhance the vertical ion convergence driven by a wind/wind shear mechanism basically responsible for the $E_{\mathrm{s}}$ layer formation. An upward directed 
electric field can disrupt the vertical ion convergence while a downward electric field can enhance it (see Abdu et al., 2003; Carrasco et al., 2007 for further details). The vertical structure of the evening vertical electric field over the equator, associated with the EPE development, as modeled by Haerendel et al. (1992), is consistent with the vortex plasma flow as measured by the Jicamarca Radar (Kudeki and Battacharyya, 1999) in the example shown in Fig. 3. The local time of the vortex center could vary from one evening to another. While it occurred at $\sim 1920$ LT in Fig. 3, the modeling by Haerendel et al. (1992), was done for $1900 \mathrm{~T}$. The vertical structure through the vortex center consists of an upward directed field in the height region $\sim 90-300 \mathrm{~km}$ and a downward directed field above $\sim 300 \mathrm{~km}$. When this electric field is field-line mapped to off-equatorial latitudes, we have an upward directed electric field over Fortaleza $\left(3.9^{\circ} \mathrm{S}, 38.45^{\circ} \mathrm{W}\right.$, dip angle: $\left.-9^{\circ}\right)$ and a downward directed electric field at a location farther away in latitude, such as Cachoeira Paulista. As explained by Abdu et al. (2003), this situation leads to disruption of $E_{\mathrm{s}}$ layer formation over Fortaleza while uninterrupted, or even enhanced, $E_{\mathrm{s}}$ layer formation over Cachoeira Paulista during the EPE development. Results in support of this explanation were presented in Fig. 8 of Abdu et al. (2003). More recently, a numerical simulation of the $E_{\mathrm{s}}$ layer evolution in the evening hours was performed by Carrasco et al. (2007) using an $E$ layer model that included all important molecular and metallic ions and electrodynamical and photo-chemical processes. Continuity and momentum equations, including the effect of the vertical electric field, were solved for the different species to obtain the $E$ layer electron density vertical profiles. The vertical structure of the vertical electric field used in the solution was obtained from an $E-F$ region electrical coupling model (Heelis et al., 1974; Batista et al., 1986) adapted for the Brazilian longitude (see Carrasco et al., 2007 for details). Figure 10 (taken from Carrasco et al., 2007) shows in the left panel the results obtained for Fortaleza when no electric field was included in the calculation and the $E_{\mathrm{s}}$ layer continues to be strong during the hours following sunset extending to midnight. On the other hand, the result plotted in the right panel was obtained by including the vertical electric field in the calculation, which shows $E_{\mathrm{s}}$ layer disruption starting at $\sim 20 \mathrm{LT}$ and its reconstitution after about $2 \mathrm{~h}$. The electric field was directed upward (corresponding to westward plasma flow) in the $F$ layer bottom-side until $\sim 300 \mathrm{~km}$ and downward directed above that height (eastward plasma flow), as depicted in Fig. 3. The upward directed electric field is responsible for the $E_{\mathrm{s}}$ layer disruption over Fortaleza simulated in Fig. 10. The downward directed electric field at higher heights maps to higher latitudes, such as Cachoeira Paulista, where the $E_{\mathrm{s}}$ layer should continue uninterrupted or enhanced, as was indeed observed (Abdu et al., 2003). Note that the case of significant EPE that causes the $E_{\mathrm{s}}$ layer disruption correspond to the conditions propitious for the ESF development. This situation can result in an anti-correlated variation between the post-sunset ESF and $E_{\mathrm{s}}$ layer occurrences at latitudes closer to the equator (such as Fortaleza) but a positive correlation between them for latitudes farther away from the equator, such as Cachoeira Paulista (Abdu et al., 2006b). Therefore, this relationship appears to clarify the negative correlation between ESF and $E_{\mathrm{s}}$ layer occurrences reported for the equatorial station Jicamarca by Stephan et al. (2002) as opposed to the positive correlation between them reported for a low latitude site Chug-Li by Bowman and Mortimer (2003).

\section{Discussion}

The EPE, $E_{\mathrm{s}}$ layer and ESF phenomena are interconnected in additional ways by the $E$ layer conductivity distribution in the evening hours. The field line integrated conductivities $\left(\Sigma_{P}\right.$ and $\left.\Sigma_{H}\right)$ and their local time/longitude gradients $\left(\Delta \Sigma_{P}\right.$ and $\left.\Delta \Sigma_{P}\right)$ are known to control/influence the EPE and ESF development. Thus, $E_{\mathrm{s}}$ layers, present at the feet of the related field lines and which may possibly enhance the integrated conductivities, could modify the developments of the EPE and ESF. An evaluation of the $E_{\mathrm{s}}$ layer contribution to the conductivity parameters is, however, a challenging task due to the limited horizontal extension of these layers and their heights in the evening hours being generally well below the height of the Pederson conductivity peak near $135 \mathrm{~m}$ (large majority of the $E_{\mathrm{s}}$ layers are located approximately near $110 \mathrm{~m}$ in the evening hours). Stephan et al. (2002) have shown that an increase of integrated conductivity attributed to a presence of sporadic $E$ layers could reduce the Rayleigh-Taylor instability growth rate and, hence, negatively influence the ESF development. A general enhancement in the field line integrated conductivity in the evening hours can cause a reduction in its gradient across the terminator. Such a reduction in the longitudinal/local time gradient in the conductivity can reduce the amplitude of the EPE, as can be verified from the modeling results presented in Fig. 2 wherein the amplitude of the EPE can be seen to increase (decrease) with increase (decrease) in the conductivity $\left(\Delta \Sigma_{P}\right)$ local time gradient in the evening. A more detained modeling by Carrasco et al. (2005) considered different latitudinal ranges/bins of enhanced conductivity, and it was found that conductivity enhancement in a latitude range $6^{\circ}-8^{\circ}$ has the largest effect on the EPE. The $E$ layer field lines at this latitude range have an apex height of 200-250 km, wherein the electron density has large gradient at the time of the maximum in EPE development. It appears, therefore, that the sporadic $E$ layers possibly contributing to conductivity increases may produce the largest effect on the EPE when they occur in this latitude range more than when they occur anywhere else. Irrespective of whether the $E_{\mathrm{s}}$ layer contribution to conductivity increase is large enough, it is worthy of note here that this latitude bin falls within the latitude range extending to either side of the dip equator wherein the $E_{\mathrm{s}}$ layer can be disrupted by the upward directed electric field of a developing EPE discussed before (see also Abdu et al., 2003). It was suggested by Abdu et al. (2003) that the latitude of the post sunset $E_{\mathrm{s}}$ layer disruption may extend up to $\pm 10^{\circ}$ depending upon the height of the nodal point (zero electric field) of the vortex flow over the equator. Indeed, the results from a conjugate point equatorial experimental (COPEX) campaign (Abdu et al., 2007; Batista et al., 2007) showed the coexistence of $E_{\mathrm{s}}$ layers of significant intensity and spread $F$ in the ionograms at the conjugate sites lo- 

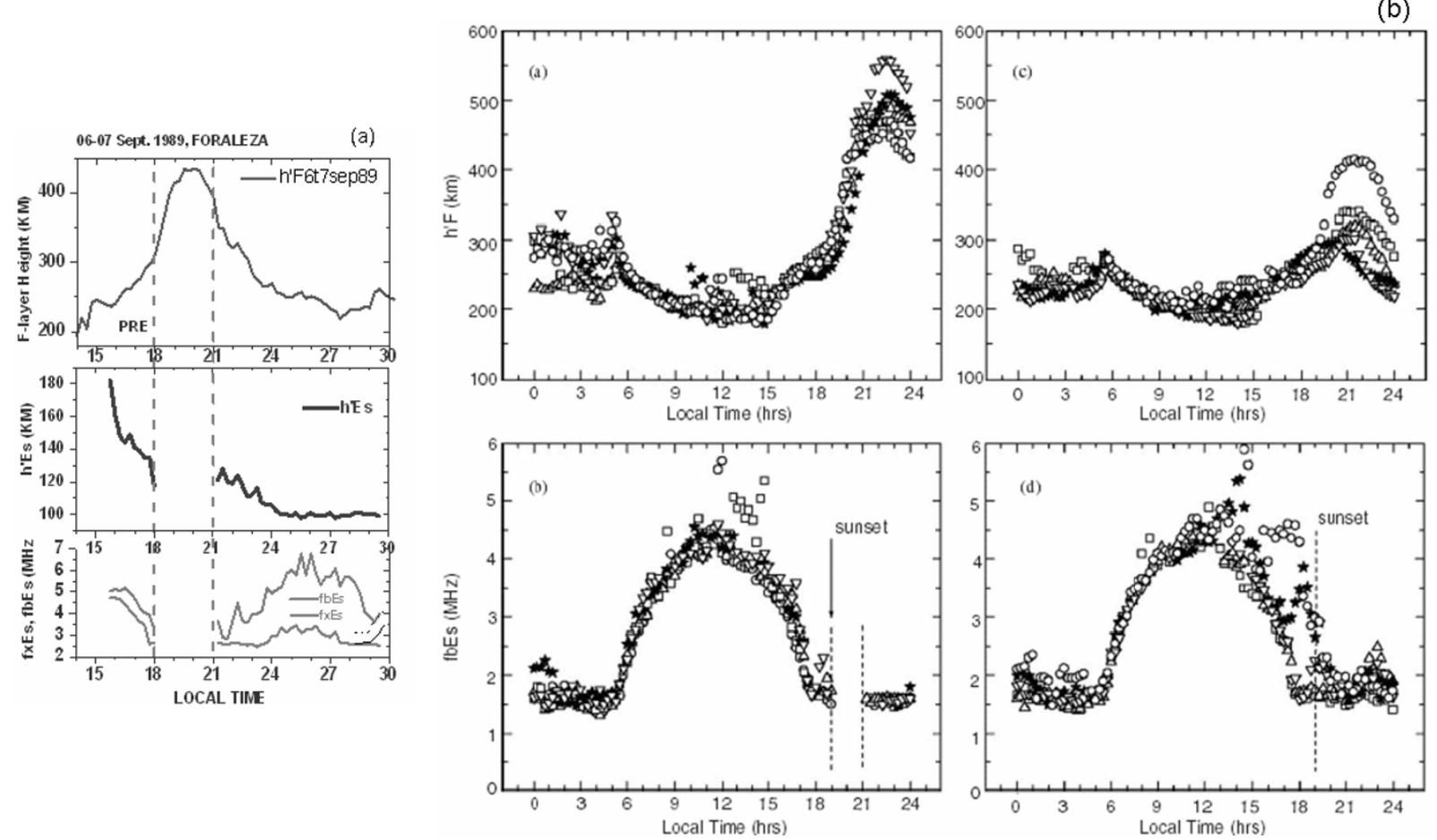

Fig. 9. (a) $F$ layer base virtual height $\left(h^{\prime} F\right)$ variation (top panel) during the evening to night hours on 6-7 September 1989; the corresponding h'Es variation (middle panel) the top frequency of the extra ordinary trace of the $E_{\mathrm{s}}$ layer $f_{\mathrm{x}} E_{\mathrm{s}}$ and the blanketing frequency $f_{\mathrm{b}} E_{\mathrm{s}}$ (bottom panel). (b) Diurnal variation of $F$ layer virtual height $h^{\prime} F$ and sporadic $E$ layer blanketing frequency $\left(f_{\mathrm{b}} E_{\mathrm{s}}\right)$ for two groups of days, one group representing a large $F$ layer height increase at sunset accompanied with post sunset disruption of sporadic $E$ layer (left panels) and another group representing weaker $F$ layer uplift for which the $E_{\mathrm{s}}$ layer disruption did not occur. The data is for June 2001 .
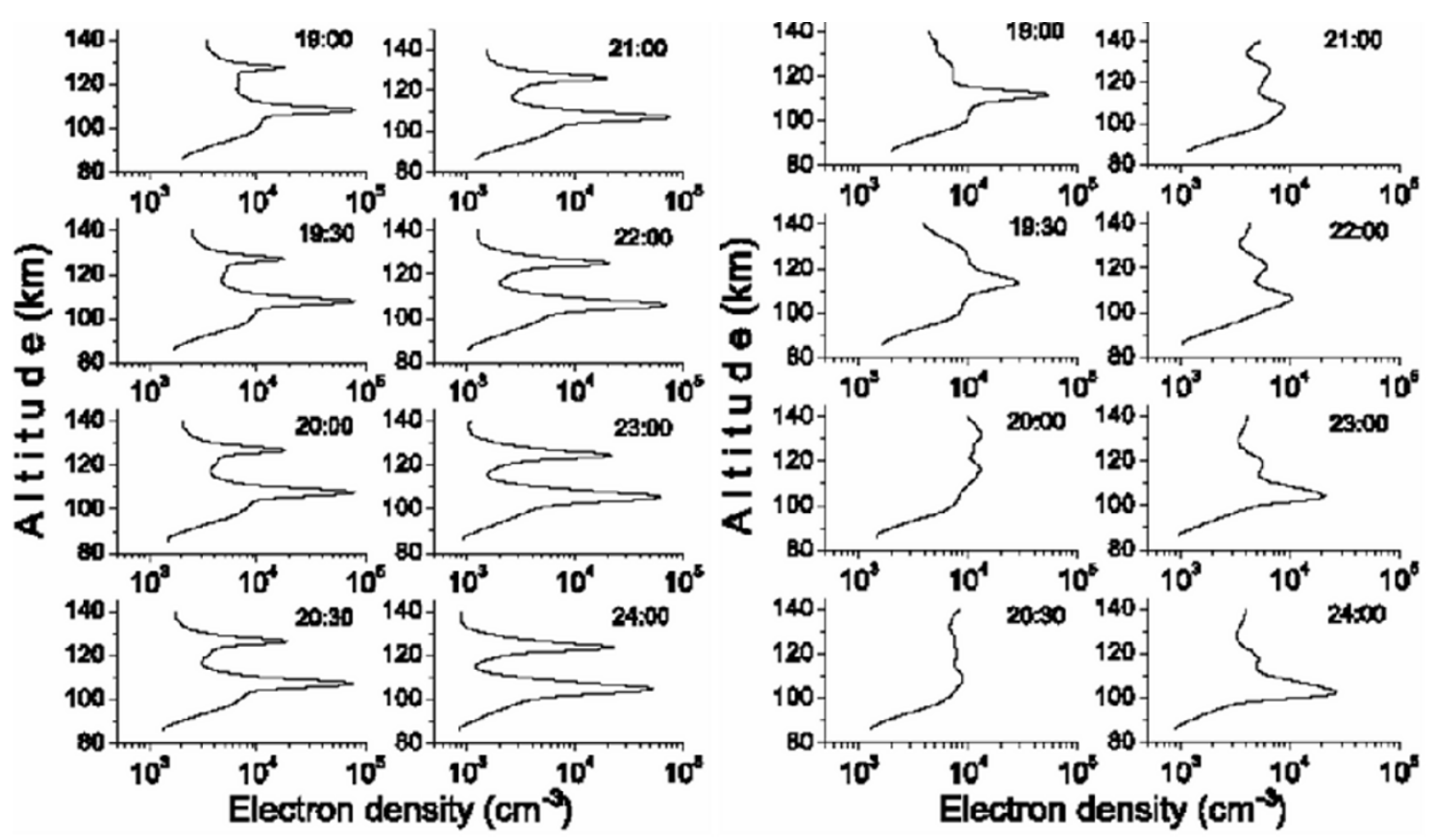

Fig. 10. E layer electron density profiles obtained from model calculation by Carrasco et al. (2007) that shows the $E_{\mathrm{s}}$ layer evolution by wind shear mechanism during the evening hours from $18 \mathrm{~T}$ to $24 \mathrm{~T}$. Left panel shows the persistence of the $E_{\mathrm{s}}$ layer when vertical electric field is neglected in the calculation. The inclusion of vertical electric field resulted in total disappearance of the $E_{\mathrm{S}}$ layer starting at $20 \mathrm{~T}$, and the restructuring the $E_{\mathrm{S}}$ layer can be noted starting at $\sim 22 \mathrm{LT}$.

cated at $\pm 12^{\circ}$ from the dip equator, as expected. On the other hand, it has been shown that EPE of the sufficient amplitude that can disrupt the $E_{\mathrm{s}}$ layers (just mentioned above) can also cause ESF development, and thus we have a situ- ation in which the ESF and $E_{\mathrm{s}}$ layers may not coexist during the early post-sunset hours (as also mentioned above). The interactive connections among the $E_{\mathrm{s}}$ layers, EPE and ESF based on the above reasoning are shown schematically 
in Fig. 11. This figure indicates (a) the EPE as a main cause of ESF, (b) the EPE, through its associated vertical electric field, as contributing to $E_{\mathrm{s}}$ layer disruption when this electric field is upward directed and (c) the $E_{\mathrm{s}}$ layers, or possibly equivalently the $\sum_{P}$ and $\Delta \sum_{P}$, as exercising more likely negative influences on both the EPE and ESF. (It should be pointed out here that the conductivity gradient due an $E_{\mathrm{s}}$ layer may cause a decrease or even enhancement of the EPE intensity depending upon the sense in which the longitudinal gradient in $E$ layer conductivity across the sunset terminator gets modified by its presence). We must keep in mind, however, that the interactive connection depicted in this figure is latitude dependent within certain latitude region centered on the dip equator. The role of PWs in the day-to-day variability of the EPE and, hence, of ESF appears to be relatively somewhat better perceivable in terms of the concerned interactive processes than is the case with the role of $E_{\mathrm{s}}$ layers in such variability. In the former case, the important questions concern the connection between the PW oscillations in winds at mesospheric heights and the EPE, which involves considerations on the height regions in the ionosphere up to which the PWs could propagate. Model studies have been conducted on the vertical propagation characteristics of these waves (see, for example, Hagen et al., 1993; Miyoshi and Hirooka, 1999). Due to the long vertical wavelength $(>50 \mathrm{~km})$, the UFK waves could penetrate into the mesosphere and even above $100 \mathrm{~km}$, as pointed out by Forbes (2000). As regards the more general classes of planetary waves, there currently appears to be theoretical difficulties in presenting their upward propagation to ionospheric heights (Hagen et al., 1993) so that their ionospheric manifestations have been attributed to the electrodynamic signatures of the PW modulated tidal modes interaction with the dynamo region (lower $E$ region) of the ionosphere. In fact, evidence on the influence of PW-modulated atmospheric tides of diurnal and semidiurnal periodicities on midlatitude sporadic $E$ layer formation has been provided by Pancheva et al. (2003). Over the equatorial region, the generation of the EPE by $E$ - and $F$ region electrodynamical coupling processes makes it necessary that the PW effects reaching at least the $E$ layer heights be the main cause of the oscillations observed in $V_{\mathrm{zp}}$. It was pointed out earlier that the two main factors that shape the EPE intensity are (1) the thermospheric zonal wind, which is eastward in the evening and (2) the longitudinal/local time gradient in the $E$ region integrated conductivity. The EPE development has been explained by Rishbeth (1971) as arising from the curl-free conditions applied to the rapidly changing (from day-to night-side) evening $F$ region vertical electric field as given by:

$$
E_{z}=U_{y} \times B_{0}\left[\Sigma_{F} /\left(\Sigma_{F}+\Sigma_{E}\right)\right]
$$

where $U_{y}$ is the thermospheric zonal wind, $B_{0}$ is the geomagnetic field intensity and $\sum_{E}$ and $\sum_{F}$ are the integrated conductivities, respectively, of the $E$ - and $F$ - regions (Abdu et al., 2006b). Due to the faster post-sunset decay of $\sum_{E}$, as compared to $\sum_{F}, E_{z}$ tends to increase towards the nightside, and the application of curl-free condition to such an electric field could lead to the enhanced zonal electric field, as originally proposed by Rishbeth (1971). It is not known

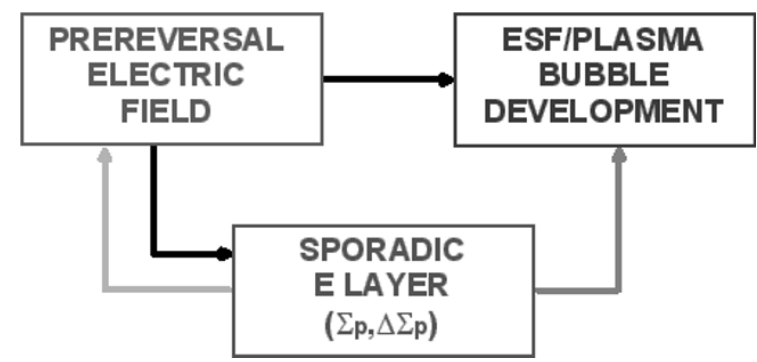

Fig. 11. A schematic of the relationship between the EPE, ESF and $E_{\mathrm{s}}$ layers in the evening hours. Note the interactive relationship between the EPE and $E_{\mathrm{S}}$ layer.

if the thermospheric winds could be modulated by the PWs, which in general do not propagate to such large heights. On the other hand, the amplitude of the EPE is very sensitive to the longitudinal/local time gradient in the $\sum_{E}$ (Abdu et al., 2004), which is determined by the post sunset ionization decay by recombination and the vertical plasma transport by tidal winds (possibly modulated by planetary waves as stated before). In the recent study by Abdu et al. (2006a), it was shown that changes in the amplitude and phase of diurnal and semi-diurnal tidal modes can cause change in the conductivity longitudinal gradient that appears to be significant enough to modify the EPE amplitude. In this way, these results appear to provide evidence on the key role of the $E$ layer conductivity, that is, the evening conductivity longitudinal/local time gradient for the discussed case, in the upward coupling of the PW effects into the equatorial $F$ region.

As a further step towards verifying the role of the $E$ layer conductivity, some additional results are presented in Fig. 12 wherein the analyzed data corresponds to the same period and site as in Fig. 5. The $h F$ values at a plasma frequency of $4 \mathrm{~Hz}$ during post sunset and post-midnight intervals, taken as the mean of the values during 21-23 T (19-21 T) and during 05-07 T (02-05 T), respectively, for Campo Grande, are plotted in the top panel of Fig. 11 with their wavelet power spectra plotted in the lower panels. The post-sunset $F$ layer heights $(h F)$ can be considered to be integrated values of the evening $V_{z}$ as given by: $h F(t)=h_{0} F+\int V_{\mathrm{z}} d t$; here $h_{0} F$ corresponds to the starting height at approximately at $17 \mathrm{~T}$, which is just prior to the onset of the evening height enhancement. The integration should be valid as long as the $h F(t)$ continues to be $\geq 300 \mathrm{~m}$, usually till around midnight for the Brazilian region. Therefore, the wavelet spectral analysis for the EPE and post-sunset $F$ layer height should yield similar results. This appears to be nearly the case when we compare the results at $21-23 \mathrm{~T}$ in Fig. 12 with the $V_{\text {zp }}$ results for Campo Grande in Fig. 5. The PW oscillations of similar periods (3-7 days) during the same day interval (around day 305) are evident in both parameters. On the other hand, the result for $h F$ during the post midnight (05-07 T) interval does not show any corresponding PW oscillation, which appears to be a significant result. The reason for the PW oscillation not being present at these hours may be attributed to the fact that the EPE contribution to the $h F$ does not extend to post-midnight hours. Note that the EPE intensity depends 


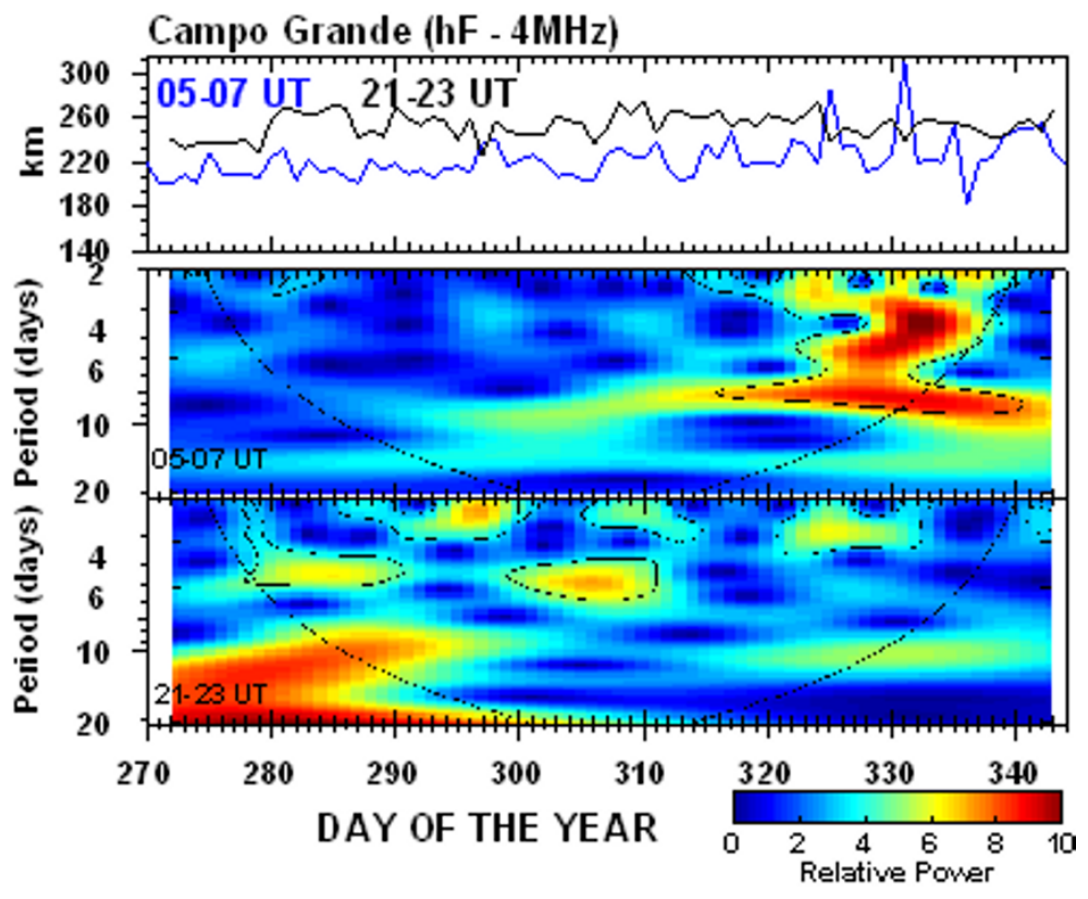

Fig. 12. The $F$ layer height $(h F)$ at $4 \mathrm{MHz}$ as mean values during the intervals 05-07 T (02-04 T) and 21-23 T (19-21 T) over Campo Grande plotted from day 270 to day 343 (October-December period) of 2002 (top panel); the wavelet power spectra of the $h F$ values during the two local time intervals respectively in the middle and bottom panels.

upon the $E$ layer conductivity local time gradient. This result would thus suggest that $E$ layer conductivity longitudinal/local time gradient is a connecting link between the upward propagating PWs and oscillations in the EPE and post sunset $F$ layer height.

\section{Conclusions}

In this paper we have discussed some important aspects of the vertical coupling processes of the equatorial atmosphere-ionosphere system that play key roles in the widely observed day-to-day variability of the evening prereversal electric field enhancement/vertical plasma drift that has consequences on the spread $F$ /plasma bubble developments. We focused on the effects of upward propagating planetary waves on the EPE and ESF and on the interactive role of evening sporadic $E$ layers in them. The main conclusions of this paper (supported by other recent results referred to in this paper) may be summarized as follows: large day-to-day variation in the evening prereversal electric field and spread $F$ can be caused by upward propagating planetary waves; sunset $E$ layer conductivity, with its longitudinal/local time gradient, modulated by the $E$ layer winds plays a leading role in the variability of the EPE and postsunset $F$ layer heights that account for part of the ESF variability (the planetary wave modulation of the tidal modes appears to be responsible for the variability in the $E$ layer winds); the role of evening sporadic $E$ layers in the EPE and ESF appears to operate in interactive ways and is dependent on the latitude of their occurrence, closer to the dip equator their development being negatively influenced by the EPE. This relationship and especially the interactive role of the $E_{\mathrm{s}}$ layers from latitude farther away form the dip equator needs to be evaluated from analysis of more data.
Acknowledgments. The authors wish to acknowledge the supports from the FAPESP through the projects 1999/00437$0,04 / 07695-5$ and $05 / 52654-8$ and $\mathrm{CNPq}$ through grants no 520185/95-1 and 502902/2007-8.

\section{References}

Abdu, M. A., Major phenomena of the equatorial ionosphere thermosphere system under disturbed conditions (Invited review), J. Atmos. Terr. Phys., 59(13), 1505-1519, 1997.

Abdu, M. A., Outstanding problems in the equatrial ionospherethermosphere system relevant to spread F, J. Atmos. Sol.-Terr. Phys., 63, 869, 2001.

Abdu, M. A., R. T. Medeiros, J. A. Bittencourt, and I. S. Batista, Vertical ionization drift velocities and range type spread $F$ in the evening equatorial ionosphere, J. Geophys. Res., 88, 399-402, 1983.

Abdu, M. A., I. S. Batista, H. Takahashi, J. MacDougall, J. H. Sobral, A. F. Medeiros, and N. B. Trivedi, Magnetospheric disturbance induced equatorial plasma bubble development and dynamics: A case study in Brazilian sector, J. Geophys. Res., 108(A12), 1449, doi:10.1029/ 2002JA009721, 2003.

Abdu, M. A., I. S. Batista, B. W. Reinisch, and A. J. Carrasco, Equatorial $F$-layer heights, evening prereversal electric field, and night $E$ layer density in the American sector: IRI validation with observations, $A d v$. Space Res., 34, 1953-1965, 2004.

Abdu, M. A., P. P. Batista, I. S. Batista, C. G. M. Brum, A. J. Carrasco, and B. W. Reinisch, Planetary wave oscillations in mesospheric winds, equatorial evening prereversal electric field and spread $F, J$. Geophys. Res., 33, L07107, doi:10.1029/1005GL024837, 2006a.

Abdu, M. A., T. K. Ramkumar, I. S. Batista, C. G. M. Brum, H. Takahashi, B. W. Reinisch, and J. H. A. Sobral, Planetary wave signatures in the equatorial atmosphere-ionosphere system, and Mesosphere- $E$ - and $F$ region coupling, J. Atmos. Sol.-Terr. Phys., 68, 509-522, 2006b.

Abdu, M. A., K. N. Iyer, R. T. de Medeiros, I. S. Batista, and J. H. A. Sobral, Thermospheric Meridional Wind Control of Equatorial Spread F and Evening Prereversal Electric Field, Geophys. Res. Lett., 2006c (in press).

Abdu, M. A., I. S. Batista, B. W. Reinisch, J. R. de Souza, J. H. A. Sobral, F. Bertoni, T. R. Pedersen, A. F. Medeiros, N. J. Schuch, and E. R. de Paula, Conjugate Point Equatorial Experiment (COPEX) Campaign in Brazil: Electrodynamics highlights related to spread $F$ development conditions and its day-to-day variability, J. Atmos. Sol.-Terr. Phys., 2007 
(submitted).

Batista, I. S., M. A. Abdu, and J. A. Bittencourt, Equatorial $F$-region vertical plasma drifts: seasonal and longitudinal asymetries in the American sector, J. Geophys. Res., 91, 12055-12064, 1986.

Batista, I. S., M. A. Abdu, A. J. Carrasco, B. W. Reinisch, E. R. de Paula, N. J. Schuch et al., Equatorial spread $F$ and sporadic $E$-layer connections during the Brazilian Conjugate Point Equatorial ExperimentCOPEX, J. Atmos. Sol.-Terr. Phys., 2007 (submitted).

Bittencourt, J. A. and M. A. Abdu, A theoretical comparison between apparent and real vertical ionization drift velocities in the equatorial $F$ region, J. Geophys. Res., 86, 2451-2455, 1981.

Bowman, G. G. and I. K. Mortimer, Spread- $F$ /sporadic E coupling at Chung-Li, especially for postsunset periods of sunspot maximum years, J. Geophys. Res., 108(A4), 1148, doi:10.1029/2002JA009541, 2003.

Carrasco, A. J., I. S. Batisat, and M. A. Abdu, The prereversal enhancement in the vertical drift for Fortaleza and sporadic $E$ layer, J. Atmos. Sol.-Terr. Phys., 67, 1610-1617, 2005.

Carrasco, A. J., I. S. Batista, and M. A. Abdu, Simulation of the sporadic $E$ layer response to prereversal associated evening vertical electric field enhancement near dip equator, J. Geophys. Res., 112, A06324, doi:10.10292006JA01243, 2007.

Chen, P. R., Two-day oscillations of the equatorial ionization anomaly, $J$. Geophys. Res., 979(A5), 6343-6357, 1992.

Eccles, J. V., A simple model of low-latitude electric fields, J. Geophys. Res., 103, 26,699-26,708, 1998.

Farley, D. T., B. B. Balsley, and R. F. Woodman, Equatorial spread FImplications VHF radar observations, J. Geophys. Res., 75(34), 7199, 1970.

Farley, D. T., E. Bonelli, B. G. Fejer, and M. F. Larsen, The prereversal enhancement of the zonal electric field in the equatorial ionosphere, $J$. Geophys. Res., 91, 13,723-13,728, 1986.

Fejer, B. G., L. Scherliess, and E. R. de Paula, Effects of the vertical plasma drift velocity on the generation and evolution of equatorial spread $F, J$. Geophys. Res., 104, 19,859-19,870, 1999.

Fesen, C. G., G. Crowley, R. G. Roble et al., Simulation of the pre-reversal enhancement in the low latitude vertical ion drifts, Geophys. Res. Lett., 27(13), 1851-1854, 2000.

Forbes, J. M., Planetary waves in the thermosphere-ionosphere system, $J$. Geomag. Geoelectr., 48, 91, 1996.

Forbes, J. M., Wave coupling between the lower and upper atmosphere: case study of an ultra-fast Kelvin Wave, J. Atmos. Sol.-Terr. Phys., 62, 1603-1621, 2000.

Forbes, J. M. and S. Leveroni, Quasi16-day oscillations in the ionosphere, Geophys. Res. Lett., 19, 981-984, 1992.

Fukao, S., Coupling Processes in the Equatorial Atmosphere (CPEA): a project overview, J. Meteor. Soc. Jpn., 84A, 1-18, 2006.

Fukao, S., T. Yokoyama, T. Tayama et al., Eastward traverse of equatorial plasma plumes observed with the Equatorial Atmosphere Radar in Indonesia, Ann. Geophys., 24(5), 1411-1418, 2006.

Fritts, D. C., M. A. Abdu, B. R. Batista, I. S. Batista, P. P. Batista, R. Buriti, B. R. Clemesha, T. Dautermann, E. de Paula, B. J. Fechine, B. Fejer, D. Gobbi, J. Haase, F. Kamalabadi, B. Laughman, L. M. Lima, H.-L. Liu, A. Medeiros, P.-D. Pautet, D. M. Riggin, F. São Sabbas, J. H. A. Sobral, P. Stamus, H. Takahashi, M. J. Taylor, S. L. Vadas, and C. M. Wrasse, The Spread $F$ Experiment (SpreadFEx): Program overview and first results, Earth Planets Space, 61, this issue, 411-430, 2009.

Gurubaran, S., S. Sridharan, T. K. Ramkumar, and R. Rajaram, The mesospheric quasi 2-day wave over Tirunelveli, J. Atmos. Terr. Phys., 63, 975-985, 2001.

Hagan, M. E., J. M. Forbes, and F. Vial, Numerical investigation of the propagation of the quasi-two-daywave into the lower thermosphere, $J$. Geophys. Res., 98(D12), 23,193-23,205, 1993.

Haerendel, G., Theory of equatorial spread F, report, Maxplanck-Institut fur Extraterre. Phys., Garching, Germany, 1973.

Haerendel, G. and J. V. Eccles, The role of the equatorial electrojet in the evening ionosphere, J. Geophys. Res., 97, 1224-1243, 1992.

Haerendel, G., J. V. Eccles, and S. Kir, Theory of modeling the equatorial evening ionosphere and the origin of the shear in the Horizontal plasma flow, J. Geophys. Res., 97, 1209-1223, 1992.

Haldoupis, C., D. Pancheva, and N. J. Mitchell, A study of tidal and planetary wave periodicities present in midlatitude sporadic $E$ layers,
J. Geophys. Res., 109, A02302, doi:10.1029/2003JA010253, 2004.

Heelis, R. A., P. C. Kendall, R. J. Moffet, D. W. Windle, and H. Rishbeth, Electrical coupling of the $E$ - and $F$-region and its effects on the $F$ region drifts and winds, Planet. Space Sci., 22, 743-756, 1974.

Hocking, W. K., B. Fuller, and B. Vandepeer, Real-time determination of meteor related parameters utilizing modern digital technology, J. Atmos. Sol.-Terr. Phys., 63(2-3), 155-169, 2001.

Hysell, D. L. and E. Kudeki, Collisional shear instability in the equatorial $F$ region ionosphere, J. Geophys. Res., 109, A11301, doi:10.1029/ 2004JA010636, 2004.

Kudeki, E. and S. Bhattacharyya, Postsunset vortex in equatorial $F$ region plasma drifts and implications for ottomside spread $F$, J. Geophys. Res., 104(A12), 28,163-28,170, 1999.

Maruyama, T., A diagnostic model for equatorial spread $F$, 1, Model description and application to electric fields and neutral wind effects, J. Geophys. Res., 93, 14,611-14,622, 1988.

Mendillo, M., J. Meriwether, and M. Biondi, Testing the thermospheric neutral wind suppression mechanism for the day-to-day variability of equatorial spread F, J. Geophys. Res., 106, 3655-3663, 2001.

Miyoshi, Y. and T. Hirooka, A numerical experiment of excitation of the 5-day wave by a GCM, J. Atmos. Sci., 56, 1698-1707, 1999.

Ogawa, T., Y. Otsuka, K. Shiokawa, A. Saito, and M. Nishioka, Ionospheric disturbances over Indonesia and their possible association with atmospheric gravity waves from the troposphere, J. Meteor. Soc. Jpn., 84A, 327-342, 2006.

Pancheva, D., N. Mitchell, R. Clark, J. Drobjeva, and J. Lastovicka, Variability in the maximum height of the ionospheric F2-layer over Millstone Hill (September 1998-March 2000); influence from below and above, Ann. Geophys., 20(11), 1807-1819, 2002.

Pancheva, D., C. Houldoupis, C. E. Meek, A. H. Manson, and N. J. Mitchell, Evidence of a role for modulated atmospheric tides in the dependence of sporadic $E$ layers on planetary waves, J. Geophys. Res., 108(A5), 1176, doi:101029/2002JA009788, 2003.

Rishbeth, H., Polarization fields produced by winds in the equatorial $F$ region, Planet. Space Sci., 19, 357-369, 1971.

Sastri, J. H., M. A. Abdu, I. S. Batista, and J. H. A. Sobral, Onset Conditions of Equatorial (Range) Spread- $F$ at Fortaleza, Brazil, During the June Solstice, J. Geophys. Res., 102(A11), 24,013-24,021, 1997.

Stephan, A. W., M. Colerico, M. Mendillo, B. W. Reinisch, and D. Anderson, Suppression of equatorial spread $F$ by sporadic E, J. Geophys. Res., 107(A2), 10,1029-10,1034, 2002.

Takahashi, H., L. M. Lima, C. M. Wrasse, M. A. Abdu, I. S. Batista, D. Gobbi, R. A. Buriti, and T. Tsuda, Evidence on 2-4 day modulation of the equatorial ionosphere h'F and mesospheric airglow emission, Geophys. Res. Lett., 32, L12102, doi: 10.1029/2004GL022318, 2005 (submitted).

Takahashi, H., C. M. Wrasse, J. Fechine, D. Pancheva, M. A. Abdu, I. S. Batista, L. M. Lima, P. P. Batista, B. R. Clemesha, N. J. Schuch, K. Shiokawa, D. Gobbi, M. G. Mlynczak, and J. M. Russell, Signatures of Ultra Fast Kelvin waves in the equatorial middle atmosphere and ionosphere, Geophys. Res. Lett., 34, L11108, doi: 10.1029/2007GL029612, 2007.

Tsunoda, R. T., Seeding of equatorial plasma bubbles with electric fields from and Es-layer instability, J. Geophys. Res., 112, A06304, doi: 10.1029/2006JA012103, 2007.

Tsunoda, R. T., R. C. Livingston, and C. L. Rino, Evidence of a velocity shear in bulk plasma motion associated with post-sunset rise of the equatorial $F$ layer, Geophys. Res. Lett., 8(7), 807-810, 1981.

Vincent, R. A., Long-period motions in the equatorial mesosphere, J. Atmos. Terr. Phys., 55, 1067-1080, 1993.

Vineeth, C., T. K. Pant, C. V. Devasia et al., Atmosphere-ionosphere coupling observed over the dip equatorial MLTI region through the quasi 16-day wave, Geophys. Res. Lett., 34(12), L12102, 2007.

Yokoyama, T., Fukao S., and Yamamoto M., Relationship of the onset of equatorial $F$ region irregularities with the sunset terminator observed with the Equatorial Atmosphere Radar, Geophys. Res. Lett., 31(24), L24804, 2004.

M. A. Abdu (e-mail: maabdu@dae.inpe.br) and C. G. M. Brum 